\title{
Functional Hypophysectomy Simply by Neck-Strangulation in the Rat: Applications for ACTH and LH Assays
}

\author{
Ryo Nakayama ${ }^{1,4, *}$, Reiko Fujiwara ${ }^{1}$, Michio Masuoka ${ }^{1,3}$, Chiharu Higashiyama ${ }^{4}$, and Takashi Nonoyama ${ }^{2}$ \\ ${ }^{1}$ former Biology Research Laboratories and ${ }^{2}$ Drug Safety Research Laboratories, Takeda Chemical Industries, Ltd., \\ Osaka 532-8686, Japan \\ ${ }^{3}$ Bioscience Center of KAC Co., Ltd., Ritto, Shiga 520-3001, Japan \\ ${ }^{4}$ Ina Research Institute, Ina, Nagano 399-4501, Japan
}

Received April 19, 2004; Accepted August 3, 2004

\begin{abstract}
The classical methods for hypophysectomy require a long training-period and at the end of each experiment, it is required to check any undue remnant of the hypophyseal tissue in the sella turcica, and there must be exclusion of such datum with the remnant from the experimental group. The present method for functional hypophysectomy by neck-strangulation in the rat is very simple to perform so that even an experimenter with little experience can become easily accustomed to the technique and obtain a number of functionally hypophysectomized preparations at any time he intends, with no need to check the remnant. The cerebral blood circulation of vertebrally dislocated rats was immediately intercepted by means of neckstrangulation and artificially respired. Fifteen minutes after intravenous injection of ACTH $(0.1-3.2 \mathrm{mU} / \mathrm{rat})$ or LH $(312.5-2500 \mathrm{ng} / \mathrm{rat})$, blood was sampled from the vena cava, and sera were examined for corticosterone or testosterone by radioimmunoassay. A linear dose-response relationship was obtained within a dose range of $0.2-1.6 \mathrm{mU} / \mathrm{rat}$ of ACTH and $312.5-$ $1250 \mathrm{ng} / \mathrm{rat}$ of LH. In the ACTH assay, parapharyngeally hypophysectomized rats were compared. It was found that the sensitivity of functionally hypophysectomized preparations was 2.72 times higher than that of the parapharyngeally hypophysectomized rats.
\end{abstract}

Keywords: hypophysectomy, neck-strangulation, ACTH, LH

\section{Introduction}

There have been two classical methods, parapharyngeal (1) and trans-auricular approaches (2) for hypophysectomy in rats, both difficult techniques. Even a dexterous individual with keen perception usually requires a long training period to secure a good hand for the operation. The parapharyngeal approach is laborious, taking much time to perform the operation, while the trans-auricular approach requires technical ingenuity.

In the ACTH assays based on corticoidogenesis, the adrenal gland rapidly decreases in sensitivity with time following hypophysectomy. This is similar to other hormonal targets $(3,4)$. Therefore, operated animals should be used for experiments as soon as possible and

*Corresponding author. 3-28-10 Daiwa-Nishi, Kawanishi City, Hyougo 666-0112, Japan

FAX:+81-72-794-1968, E-mail: fwiw8201@mb.infoweb.ne.jp at a constant time after hypophysectomy. This may be troublesome, especially on purchasing operated animals.

For the resolution of this problem, the operation techniques must be simplified enough so that anyone can easily perform hypophysectomy by his own hand at any time he intends.

The present procedure is proposed as a simplified technique for hypophyseal suppression by intercepting the cerebral blood circulation by neck-strangulation with a vinyl tube following vertebral dislocation.

\section{Materials and Methods}

This study was authorized by the Takeda Experimental Animal Care and Use Committee.

Ten-week-old male Sprague-Dawley rats (SD-JCL) weighing $360-430 \mathrm{~g}$ and Fischer rats (F-344) weighing 214-242 g from Japan CLEA Co. (Tokyo) were used 
for the ACTH and LH assays, respectively. For the comparison studies, we used parapharyngeally hypophysectomized SD-JCL (male, 10-week-old) purchased from Japan CLEA weighing 306-335 g; they were used on the next day following hypophysectomy. The animals received $\gamma$-ray irradiated food pellets and water ad libitum.

The whole ampoule content of the 3rd International Working Standard for ACTH (3rd IWS ACTH) (WHO, Nat. Inst. for Med. Res., London, UK) was dissolved in saline containing $5 \%$ bovine serum albumin (BSA, protease free; Wako Pure Chemical Industries, Ltd., Osaka), protease-inhibitor Trasylol ${ }^{\circledR} 400 \mathrm{KIE} / \mathrm{ml}$ (Bayer, Leverkusen, Germany), and $0.01 \mathrm{~N} \mathrm{HCl}$ (Wako). Highly purified rat LH (for iodinations; National Hormone \& Peptide Program (NHPP)-NIDDK, Torrance, CA, USA) was similarly dissolved in the above solution except for $\mathrm{HCl}$.

Rats were vertebrally dislocated and immediately strangulated around the neck with a well-cleansed vinyl tube, $3.1 \mathrm{~mm}$ in diameter, to intercept the blood circulation into the head. Immediately after the neckstrangulation, the rats were artificially respired with a

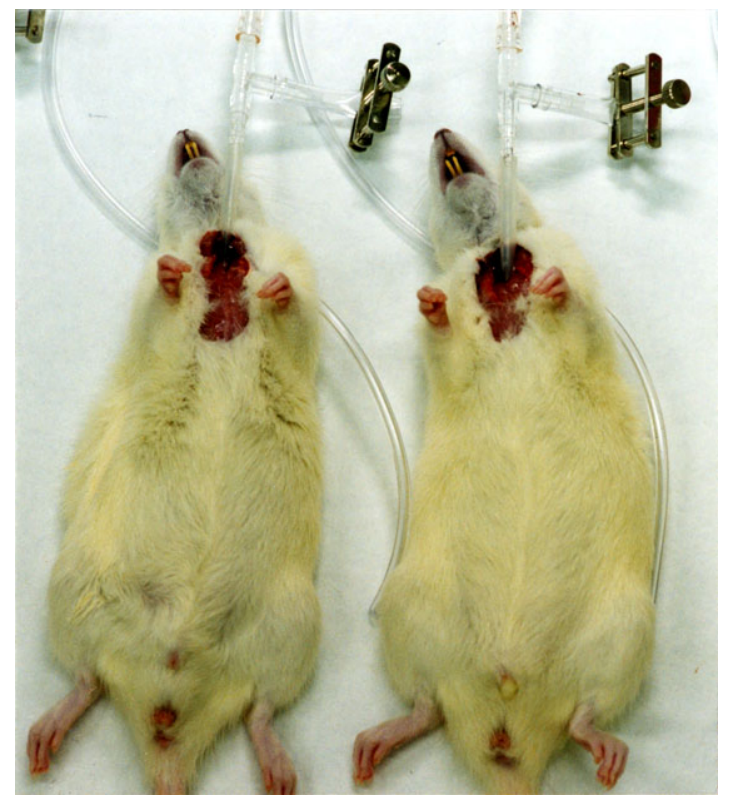

Fig. 2. View of strangulating hypophysectomized rats.

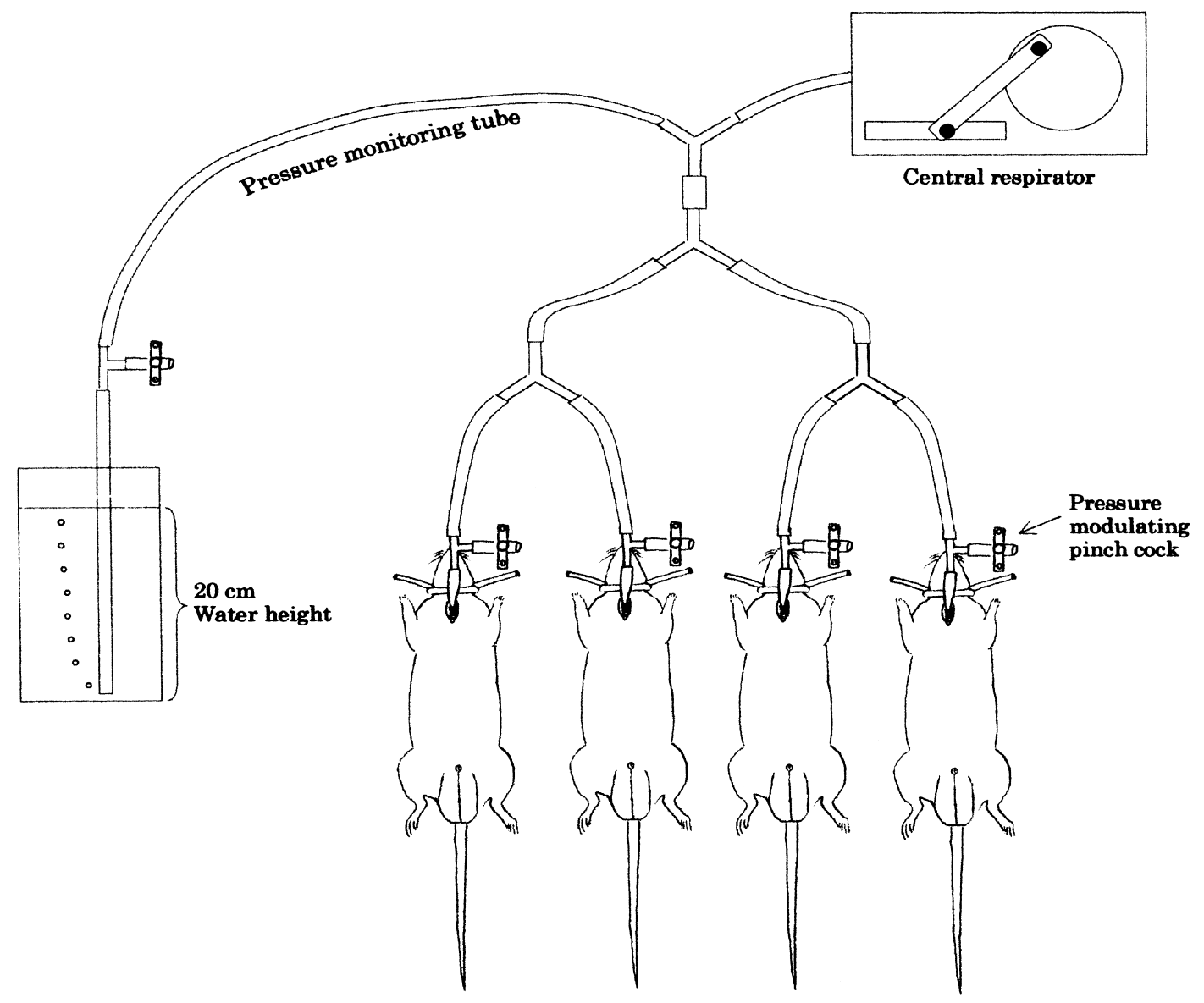

Fig. 1. Systems of strangulating hypophysectomy in the rat. 
pressure of $20 \mathrm{~cm}$ water height at a rate of 45 breaths /min using a respirator for dogs (Respirator Pump; Harvard Apparatus, Holliston, MA, USA). The whole procedure usually takes 1 or $2 \mathrm{~min}$, and the animal specimen remained alive for $6 \mathrm{~h}$ or more. Animals were strangulating-hypophysectomized in order, and side by side artificially respired using a central respirator as shown in Figs. 1 and 2. Animals for the ACTH assay were allowed to stand for $1 \mathrm{~h}$ after operation for settling down of the blood corticosterone.

ACTH or LH was injected via the tail vein, and 15 min later $(5,6)$, blood was sampled from the inferior vena cava. For the ACTH assay, blood was collected at the furcation of the left renal vein. Serum was separated and stored in a deep-freezer until radioimmunoassay using commercial kits (ICN Biomedicals, Inc, Irvine, CA, USA) for corticosterone and Dia Sorin s.r.1., (Vercelli, Italy) for testosterone.

All animal experiments were performed during 13:00 - 16:00, except the 6-h aliveness experiment.

\section{Results}

Dose-response of the serum corticosterone level to $A C T H$

Using the strangulating procedure, the dose-response was retained linear within the dose range between 0.2 and $1.6 \mathrm{mU} /$ rat of 3rd IWS ACTH, showing a regression (7) calculated as $\mathrm{Y}=463.8 \log \mathrm{X}+437.3$ (Fig. 3).
The calculated precision index $(\lambda=\mathrm{S} / \mathrm{b})$ (8) was a low value $(0.171)$, indicating that the present method is useful for reliable ACTH assay.

\section{Comparison with parapharyngeally hypophysectomized rats}

The neck-strangulated functionally hypophysectomized preparations were compared with parapharyngeally hypophysectomized rats on the next day after the operation. There was a linear response within the dose range of 0.4 and $1.6 \mathrm{mU} /$ rat of $3 \mathrm{rd} \mathrm{IWS} \mathrm{ACTH.} \mathrm{The}$ regression was calculated as $\mathrm{Y}=494.9 \log \mathrm{X}+246.7$ (Fig. 3).

In the parallel line assay, the sensitivity of the neckstrangulated functionally hypophysectomized preparations was 2.72 times higher (95\% fiducial limits $1.91-$ 4.48) than that of the parapharyngeally hypophysectomized rats.

\section{Dose-response of the serum testosterone level to $\mathrm{LH}$}

The linearity of the dose-response was also retained with LH within the dose range of $312.5-1250 \mathrm{ng} / \mathrm{rat}$ (Fig. 4). The regression and precision index $\lambda$ were calculated as $\mathrm{Y}=20.2 \log \mathrm{X}-43.8$ and 0.416 , respectively.

\section{Discussion}

In vitro bioassays for pituitary hormones are widely

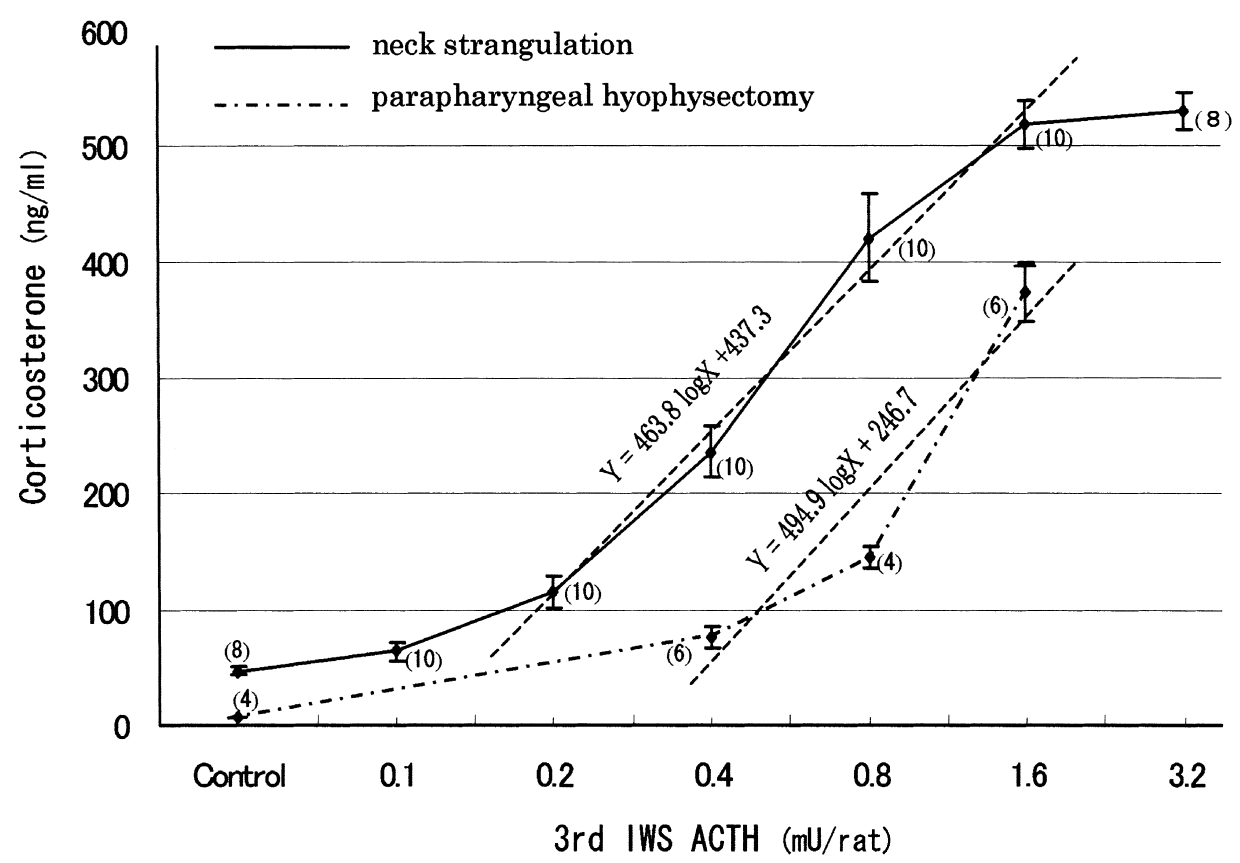

Fig. 3. Dose-responsive relationship of serum corticosterone to 3rd IWS ACTH compared with that in the parapharyngeally hypophysectomized rats. Means \pm S.E.M.; Number of rats used in parentheses. 


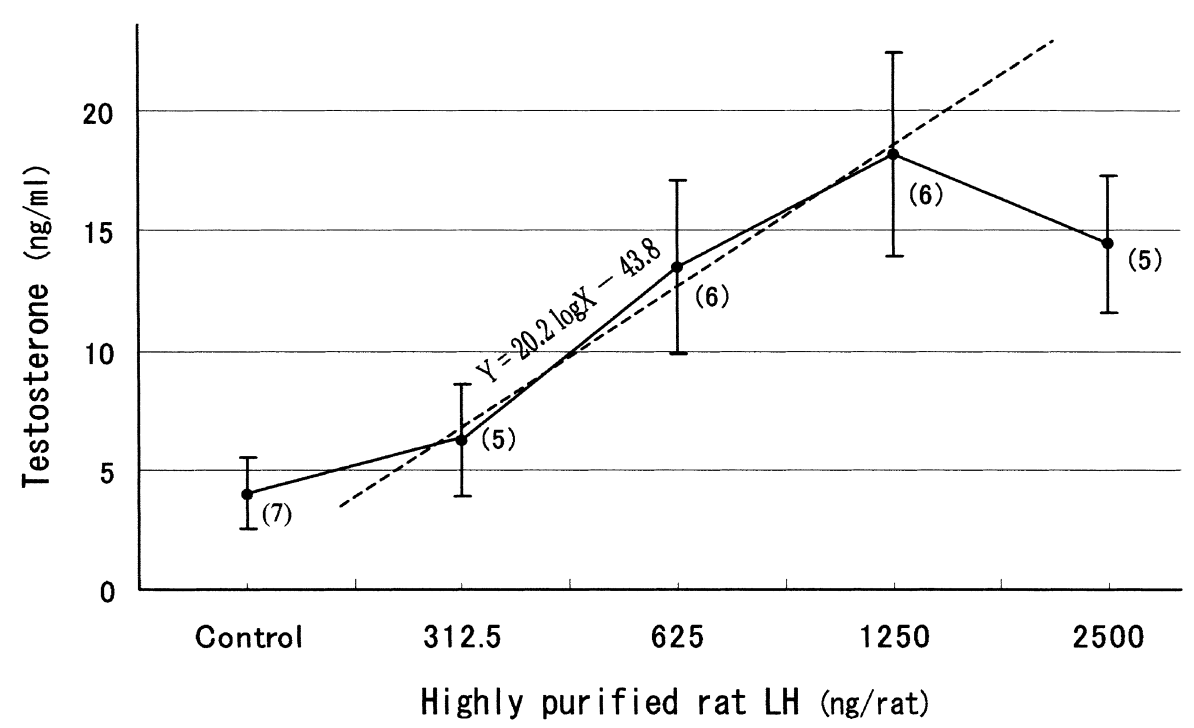

Fig. 4. Dose-responsive relationship of serum testosterone to highly purified rat LH. Means \pm S.E.M.; Number of rats used in parentheses.

adopted, but in these assays, persistency of test materials is unable to be detected, and effects of metabolisms on the activity are not taken into account. In vivo assays using hypophysectomized animals are able to detect the persistency of test materials including the effects of metabolism.

Traditional hypophysectomy is performed by the parapharyngeal (1) or trans-auricular approach (2), and the former approach provides a more complete operation, but this surgery requires much more time. On the other hand, trans-auricular approach requires respectable ingenuity for the technique. In either method, a long training-period is required for an individual to become skillful in the procedure and careful checking any undue remnants is rigidly required at the end of the experiments in the sella turcica or throughout the field operated on; data from animals containing the remnant must be excluded from the experimental group.

Target organs are known to gradually become refractory to the tropic hormones following hypophysectomy. Segaloff et al. (3) observed a very poor response in the rat ventral prostate method of LH assay two weeks after hypophysectomy, whereas the effect was much greater two days after hypophysectomy. Suzuki et al. (4) showed that in response to acutely administered hCG, testosterone was sufficiently secreted into the testicular vein in rats on the next day following hypophysectomy, but no response was seen ten days after hypophysectomy. In ACTH assays based on corticoidogenesis, a similar phenomenon was found in the present assay that prominently reduced responsiveness of the adrenal gland to ACTH occurred on the next day after parapharyngeal hypophysectomy (Fig. 3). It was shown to be lower by $1 / 2.72$ than the neck-strangulated functionally hypophysectomized preparations.

The present method is simple enough for an experimenter with little experience in this procedure to easily obtain a number of functionally hypophysectomized preparations at any time he needs them, and animals may be served at the highest in adrenal sensitivity for the assay of ACTH based on corticoidogenesis. In addition, since the blood circulation into the brain including the hypophysis is completely intercepted, there is no need for tedious checking at autopsy for the hypophyseal remnant.

In the present strangulating method, no anesthetics such as ether, phenobarbital, or morphine influencing the secretion of hypophyseal hormones such as ACTH, gonadotropin, prolactin, etc. $(9-12)$ were used. We adopted the vertebral dislocation followed by neck strangulation, since the vertebral dislocation is allowed for euthanasia under the guidelines of the American Veterinary Association Panel on Euthanasia (13).

The present procedure might be also useful for studies on ADH or oxytocin without any hypothalamic effects, because the animals hypophysectomized by the traditional method still retain their hypothalami and stalks in situ where the hormones are abundantly produced or stored.

\section{Acknowledgments}

Ethical judgement of the experimental plan by Professor Tadao Serikawa, Institute of Laboratory Animals, 
Graduate School of Medicine, Kyoto University is highly appreciated. The authors would like to express their gratitude to Dr. Hiroaki Miyajima, Chairman, Shin Nippon Biomedical Laboratories, Ltd., Kagoshima for encouraging the authors and to Drs. Toshiyuki Hamashima and Ryoetsu Imai, Drug Safety Research Laboratories of Takeda Chemical Industries, Ltd. for supporting the experiment and discussing this report with us. One of the authors, Ryo Nakayama, would like to express his gratitude to his wife, Ms. Fujiko Nakayama, for calculating the data and preparing Figs. 1, 3, and 4 using the Excel and Word programs with a personal computer. The authors acknowledge Dr. A.F. Parlow, Scientific Director, NIDDK's National Hormone \& Peptide Program for providing highly purified rat LH.

\section{References}

1 Smith PE. Hypophysectomy and a replacement therapy in the rat. Am J Anat. 1930;45:205-273.

2 Koyama R. A simple method of hypophysectomy in rats (Koyama's external auditory canal method). Endocrinol Jpn. 1962;9:321-323.

3 Segaloff A, Steelman SL, Flores A. Prolactin as a factor in the ventral prostate assay for luteinizing hormone. Endocrinology. 1956;59:233-240.

4 Suzuki Y, Eto T, Masuda H. Androgen biogenesis of testis in vivo, from progesterone- $4-{ }^{14} \mathrm{C}$ by intratesticular infusion of eviscerated adult rats; special reference to the effects of hypo- physectomy and HCG (chorionic gonadotropin) administration. Kachiku Hanshoku Kenkyu Kaishi. 1962;8:41-46. (text in Japanese with English abstract)

5 Brudieux R, Krifi MN, Laulin JP. Release of aldosterone and corticosterone from the adrenal cortex of the Brattleboro rat in response to administration of ACTH. J Endocrinol. 1986;111: 375-381.

6 Hakola K, Pierroz DD, Aebi A, Vuagnat BAM, Aubert ML, Huhtaniemi I. Dose and time relationships of intravenously injected rat recombinant luteinizing hormone and testicular testosterone secretion in the male rat. Biol Reprod. 1998;59: 338-343.

7 Nakajima Y. Forecast and intercept in regression lines. In: Excel 2000 A Handbook for Functions. Tokyo: Natsume Co; 1999. p. 273-275 and p. 348-350. (in Japanese)

8 Gaddum JH. Bioassays and mathematics. Pharmacol Rev. 1953; $5: 87-134$.

9 Ashford A, Shapero M. Effect of chlorpromazine, reserpine, benactyzine and phenobarbitone on the release of corticotrophin in the rat. Br J Pharmacol. 1962;19:458-463.

10 Briggs FN, Munson PL. Studies on the mechanism of stimulation of ACTH secretion with the aid of morphine as a blocking agent. Endocrinology. 1955;57:205-219.

11 Matsuda K, Duyck C, Kendall JW Jr, Greer MA. Pathways by which traumatic stress and ether induce increased ACTH release in the rat. Endocrinology. 1964;74:981-985.

12 Westermann EO, Maickel RP, Brodie BB. On the mechanism of pituitary-adrenal stimulation by reserpine. J Pharmacol Exp Ther. 1962;138:208-217.

13 AVMA Panel on Euthanasia. American Veterinary Medical Association. 2000 Report of the AVMA panel on euthanasia. J Am Vet Med Assoc. 2001;218:669-696. 